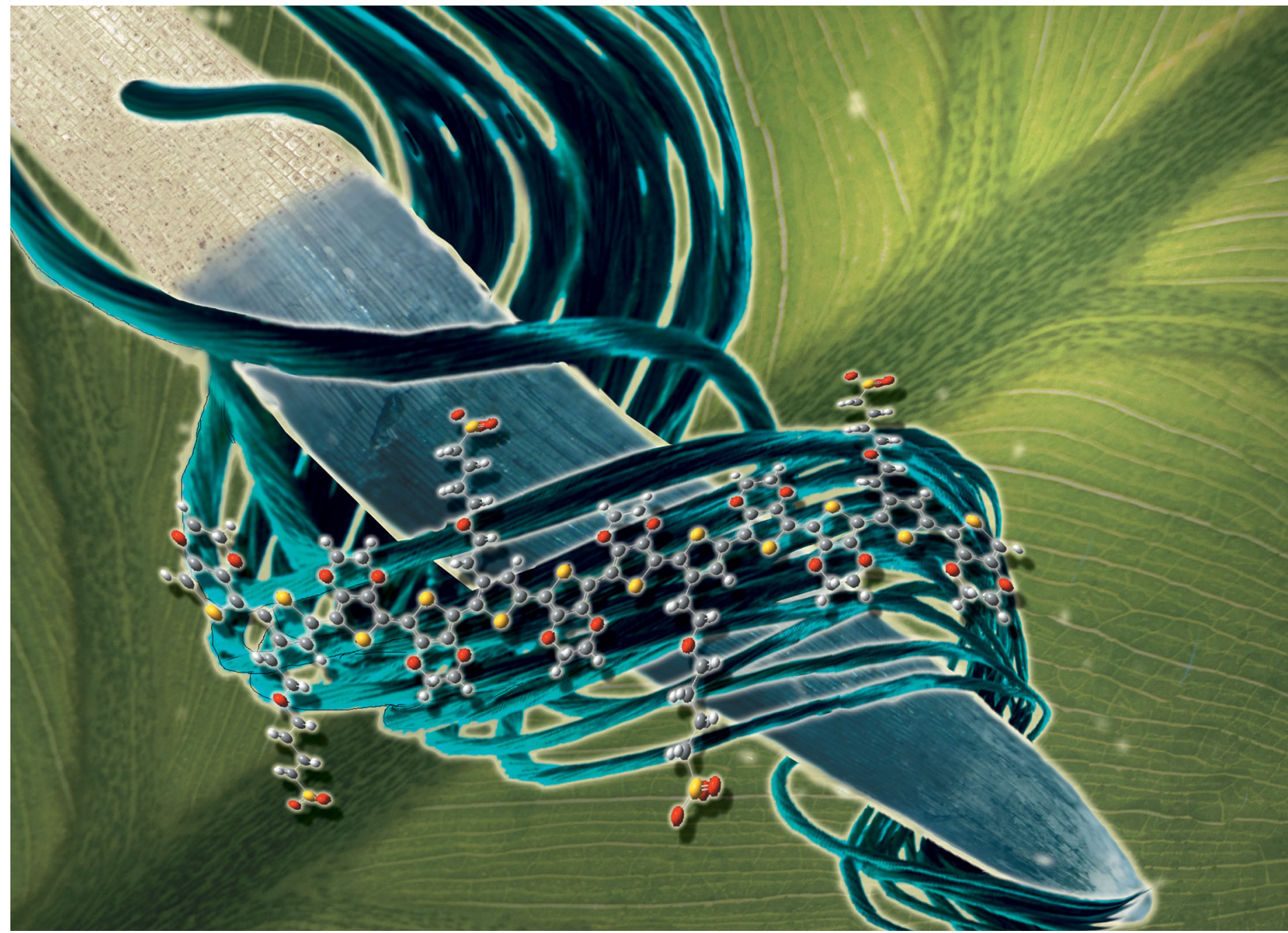

Showcasing a study on the mechanism of polymerization of conjugated oligomers and formation of conductors in vivo by Gwennaël Dufil and Dr Eleni Stavrinidou at Linköping University.

Enzyme-assisted in vivo polymerisation of conjugated oligomer based conductors

The conjugated oligomer ETE-S is enzymatically polymerized along the plant root due to the presence of peroxidase enzyme and hydrogen peroxide forming conductors integrated within the plant structure. Artwork created by Daniela Parker.

\section{As featured in:}

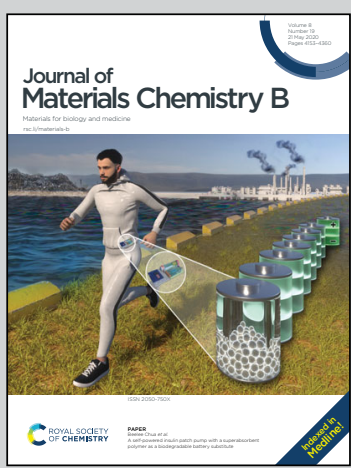

See Eleni Stavrinidou et al., J. Mater. Chem. B, 2020, 8, 4221. 
Check for updates

Cite this: J. Mater. Chem. B, 2020, 8, 4221

Received 22nd January 2020, Accepted 6th March 2020

DOI: $10.1039 / \mathrm{d} 0 \mathrm{tb} 00212 \mathrm{~g}$

rsc.li/materials-b

\title{
Enzyme-assisted in vivo polymerisation of conjugated oligomer based conductors $\dagger$
}

\author{
Gwennaël Dufil, (D) a Daniela Parker, ${ }^{a}$ Jennifer Y. Gerasimov, ${ }^{a}$ Thuc-Quyen Nguyen, ${ }^{b}$ \\ Magnus Berggren ${ }^{\mathrm{ac}}$ and Eleni Stavrinidou (D) *ac
}

Conjugated polymers conduct both electronic and ionic carriers and thus can stimulate and translate biological signals when used as active materials in bioelectronic devices. Self- and on-demand organization of the active material directly in the in vivo environment can result in the seamless integration of the bioelectronic interface. Along that line, we recently demonstrated spontaneous in vivo polymerization of the conjugated oligomer ETE-S in the vascular tissue of plants and the formation of conducting wires. In this work, we elucidate the mechanism of the in vivo polymerization of the ETE-S trimer and demonstrate that ETE-S polymerizes due to an enzymatic reaction where the enzyme peroxidase is the catalyst and hydrogen peroxide is the oxidant. ETE-S, therefore, represents the first example of a conducting polymer that is enzymatically polymerized in vivo. By reproducing the reaction in vitro, we gain further insight on the polymerization mechanism and show that hydrogen peroxide is the limiting factor. In plants the ETE-S triggers the catalytic cycle responsible for the lignification process, hacks this biochemical pathway and integrates within the plant cell wall, forming conductors along the plant structure.

\section{Introduction}

Electronic interfacing with living systems has been the main objective within the rising research area of organic bioelectronics. $^{1,2}$ Biomedical applications have been prime targets in this field, therefore much effort has been devoted to developing materials and devices that translate and stimulate ionic signalling in the biological milieu, while at the same time cause a minimal immune reaction from the target organism. Conducting polymers have been proved ideal for bio-interfacing as they support transport and close coupling between both electronic and ionic carriers, ${ }^{3}$ thus providing signal transduction between the inherently ionic biological world and human-made technology. In addition, organic chemistry offers an unlimited toolbox to build pi-conjugated systems functionalized for specific binding and interactions with the biological targets.

\footnotetext{
${ }^{a}$ Laboratory of Organic Electronics, Department of Science and Technology, Linköping University, SE-60174, Norrköping, Sweden. E-mail: eleni.stavrinidou@liu.se

${ }^{b}$ Center for Polymers and Organic Solids, Department of Chemistry and Biochemistry, Materials, and Physics, University of California, Santa Barbara, CA 93106, USA

${ }^{c}$ Wallenberg Wood Science Center, Linköping University, SE-60174, Norrköping, Sweden

$\dagger$ Electronic supplementary information (ESI) available. See DOI: 10.1039/ d0tb00212g
}

Enhancing the compatibility and integration of the bioelectronic interface has been achieved by using thin and conformable substrates or by encapsulating the rigid device within a soft matrix. Still, these strategies result in electrodes and devices that present limitations with respect to conformation and adaption to the multiscale and complex architectures of tissues. One way to overcome the limitation of substrates is to deposit the active materials directly onto the tissue. For example, Martin and co-workers demonstrated in vivo electropolymerization of poly(3,4-ethylenedioxythiophene) (PEDOT) within the brain of a mouse. ${ }^{4}$ The polymer formed a cloudlike electrode within the brain tissue without causing any major negative effects on the brain morphology and function. Along the same line, we demonstrated self-organization of PEDOTSulfonate (PEDOT-S) within the xylem vascular tissue of plants. ${ }^{5}$ The polymer was uptaken from a solution and then formed hydrogel-like conducting wires along the xylem tissue, the water transport vessels of the plant. Furthermore, we advanced the in vivo functionalization by designing a new molecule, the 4-(2-(2,5-bis(2,3-dihydrothieno[3,4- $b][1,4]$ dioxin-5-yl)thiophen-3yl)ethoxy)butane-1-sulfonate (ETE-S). ${ }^{6}$ The ETE-S molecule was not only uptaken and distributed in the vascular tissue of the plant, but, it also polymerized in vivo without the external aid of oxidants or any physical stimulation. The ETE-S based conducting wires in the vascular tissue had conductivity of $10 \mathrm{~S} \mathrm{~cm}^{-1}$, specific capacitance of $20 \mathrm{~F} \mathrm{~g} \mathrm{~g}^{-1}$ and were used as the electrodes for the construction of a supercapacitor within the plant structure. 
We hypothesized that the polymerization was driven by the natural physicochemical environment of the plant that acted as a template and catalyst for the polymerization reaction. Indeed, reactive oxygen species and enzymes are highly present in plants, but their involvement in the ETE-S polymerization has not been demonstrated.

Over the past two decades, the use of enzymes as catalysts in polymerization reactions has received significant attention as it offers a simple synthetic route at mild reaction conditions which is also environmentally benign. ${ }^{7,8}$ In vitro polymerization of conducting polymers has been achieved with the use of oxidoreductase enzymes, usually peroxidases and laccases, ${ }^{9}$ enzymes which catalyse electron transfer from electron donors to electron acceptor molecules. ${ }^{10}$ The enzymatic polymerization of aniline ${ }^{11}$ has been extensively explored but only a few studies demonstrate enzyme catalysed polymerization of Pyrrole and EDOT. Polypyrrole ${ }^{12}$ and PEDOT ${ }^{13-15}$ are successfully polymerized only at low $\mathrm{pH}$ (2). Due to the higher oxidation potential of EDOT and Pyrrole polymerization at higher $\mathrm{pH}(\sim 4)$ is possible only with the use of mediators. ${ }^{16,17}$

In this work, we attribute the in vivo polymerization of the conjugated oligomer ETE-S in plants ${ }^{6}$ to an enzymatically assisted polymerization. While conducting polymers are widely used in bioelectronics and their enzymatic polymerization has been demonstrated in in vitro conditions, the in vivo environment has not been utilized so far for the fabrication of the bioelectronic interface. ETE-S, therefore, represents the first example of a conducting polymer that is enzymatically polymerized in vivo. We demonstrate that the ETE-S molecule triggers the peroxidative cycle of the plant cell wall that is regulated by peroxidases, where upon the ETE-S polymerizes and incorporates into the plant structure. Furthermore, we investigate which parameters affect the in vitro polymerization that, to the best of our knowledge, was performed previously only in the case of polyaniline. ${ }^{18}$ Using the natural biochemical environment one can then form conductors and electronic components that are seamlessly integrated within the living tissue. Bioelectronic interfaces can thus be designed to track the native structure and to create communication pathways with external devices or augment non-native functionalities.

\section{Results and discussion}

Hydrogen peroxide and peroxidases are highly involved in the defence mechanism of the plant ${ }^{19-21}$ and therefore consist of the prime suspects with respect to catalysing the polymerization of ETE-S. Consequently, we sought to investigate, via UV-Vis absorption spectroscopy, whether the presence of $\mathrm{H}_{2} \mathrm{O}_{2}$ or peroxidase triggers the polymerization of ETE-S in vitro. An aqueous solution of ETE-S, $0.2 \mathrm{mg} \mathrm{ml}^{-1}$, was prepared and then mixed with $\mathrm{H}_{2} \mathrm{O}_{2}$ at a molar ratio of $\mathrm{H}_{2} \mathrm{O}_{2}$ vs. ETE-S equal to 0.5 and with $\mathrm{pH}$ 5-6. The solution was let to react for $30 \mathrm{~min}$ and, then, we acquired the UV-Vis absorption spectra. As seen in Fig. 1a the absorption spectra of the ETE-S solution before and after 30 min of the addition of $\mathrm{H}_{2} \mathrm{O}_{2}$ are identical indicating that there has not been any polymerization. Even if the solution of ETE-S with $\mathrm{H}_{2} \mathrm{O}_{2}$ is let to react for 15 hours still no change is observed (Fig. S1, ESI $\dagger$ ). The same results are obtained when we add peroxidase enzyme (HRP, horseradish peroxidase) to an ETE-S solution in the absence of $\mathrm{H}_{2} \mathrm{O}_{2}$. However, when both peroxidase and $\mathrm{H}_{2} \mathrm{O}_{2}$ are added to the ETE-S solution the ETE-S monomer peak diminishes and a broad absorption feature arises around $800 \mathrm{~nm}$. The feature around $800 \mathrm{~nm}$ corresponds to the doped state of the p(ETE-S) as it was previously demonstrated from our group with a combination of experimental and theoretical evidence. ${ }^{22}$ Spectroelectrochemistry performed on electropolymerized p(ETE-S) revealed that the oxidized state of the polymer corresponds to the absorption band at $800 \mathrm{~nm}$. At the same time electron paramagnetic resonance spectrometry, EPR revealed that the charge carriers in the doped state are bipolarons. The experimental evidence in that work were supported by DFT and DFT time depended calculations. ${ }^{22}$

Next, we compared the optical properties of the polymer formed via the enzymatic protocol in vitro (as described above) with the resulting material formed when ETE-S was introduced
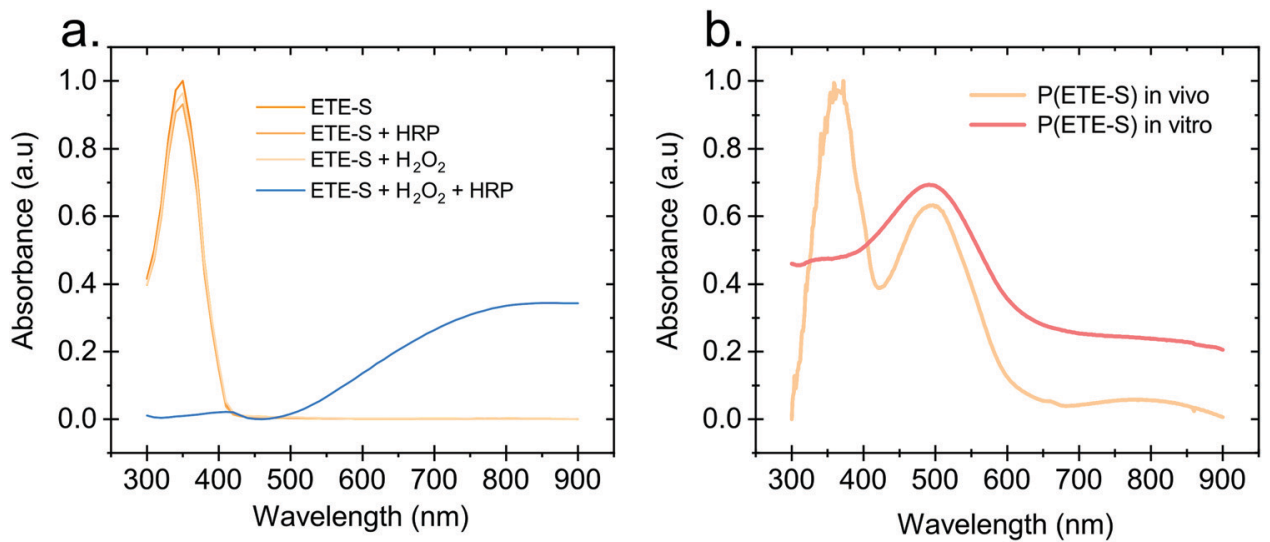

Fig. 1 In vitro polymerization of ETE-S. (a) Absorption spectra of ETE-S $(360 \mu \mathrm{M})$, ETE-S $(360 \mu \mathrm{M})$ with $\mathrm{HRP} 5 \cup \mathrm{ml}^{-1}$, ETE-S (360 $\left.\mu \mathrm{M}\right)$ with $\mathrm{H}_{2} \mathrm{O}_{2}(180 \mu \mathrm{M})$, ETE-S $(360 \mu \mathrm{M})$ with $\mathrm{H}_{2} \mathrm{O}_{2}(180 \mu \mathrm{M})$ and HRP $5 \mathrm{U} \mathrm{ml}^{-1}$. (b) Absorption spectra of DMSO extract of in vivo formed $\mathrm{p}(\mathrm{ETE}-\mathrm{S})$ in rose xylem and in vitro formed $\mathrm{p}(\mathrm{ETE}-\mathrm{S})$ with ETE-S $(360 \mu \mathrm{M}), \mathrm{H}_{2} \mathrm{O}_{2}(180 \mu \mathrm{M})$ and $\mathrm{HRP} 5 \mathrm{U} \mathrm{ml} \mathrm{l}^{-1}$. 
into the plant. Rose cuttings were immersed in ETE-S aqueous solution for 24 hours and then the functionalized xylem was collected and analysed. In order to extract the polymer formed in the plant, the functionalized xylem tissue was mechanically crushed in DMSO as previously described. ${ }^{6}$ Similarly, the polymer formed in vitro was first allowed to dry and then dissolved in DMSO. In Fig. 1b, we show the absorption spectra of the DMSO extraction of the polymer formed in vivo in the rose xylem and the polymer formed in vitro via the enzymatic protocol. In both cases, we observe a peak at $500 \mathrm{~nm}$ that corresponds to the neutral polymer with a minimum of 4 ETE-S units as defined by DFT calculations, spectroelectrochemistry and EPR. ${ }^{6,22}$ While in the in vitro formed polymer there is no presence of the monomer, in the plant extract the monomer peak is still present suggesting that the polymerization in vivo is incomplete. In addition, the polymer in both cases is mostly in its undoped form. When water is added into the polymer-DMSO solution, some polymer converts to its doped state as shown by the broad absorption feature at $800 \mathrm{~nm}$ (Fig. S2, ESI $\dagger$ ). These observations point out towards a doping mechanism that recently has been reported by Yurash et $a .^{25}$ The presence of water results in the protonation of the thiophene unit of the polymer. Upon protonation, a cation is formed in the conjugated backbone of the polymer. A neutral polymer chain can then undergo a single electron transfer with the protonated polymer giving one polymer chain with a positive polaron and another polymer chain with an unpaired electron. ${ }^{25,26}$ All the above findings suggest that the ETE-S can be polymerized with the peroxidase enzyme acting as a catalyst in the presence of hydrogen peroxide in physiological $\mathrm{pH}$.

In Fig. 2, we illustrate the proposed mechanism of ETE-S polymerization following the classical scheme of the peroxidative cycle in plants. ${ }^{27}$ Hydrogen peroxide oxidizes the peroxidase to an active enzymatic intermediate, called compound I. Compound I then oxidizes one ETE-S molecule and converts to compound II. Then, the peroxidase returns to its initial state by oxidizing a

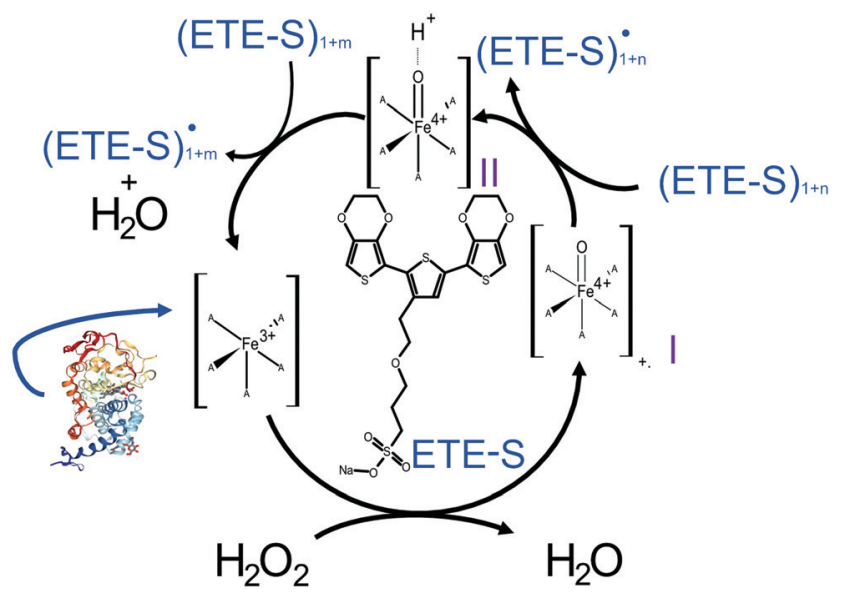

Fig. 2 Proposed mechanism of the ETE-S polymerization under the plant's peroxidative cycle with the peroxidase being the catalyst and $\mathrm{H}_{2} \mathrm{O}_{2}$ the oxidant for the polymerization reaction. Peroxidase image vizualized with NGL viewer. ${ }^{23,24}$ second ETE-S molecule and releasing water. The ETE-S radicals can then couple to form ETE-S dimers. Further polymerization can occur either by ETE-S oligomers entering the peroxidative cycle or through radical transfer between the ETE-S radical to ETE-S oligomers. The radicals then take part in oxidative coupling to form larger oligomers.

In order to gain further insight into the polymerization reaction, we investigated the effect of the relative $\mathrm{H}_{2} \mathrm{O}_{2}$ and ETE-S concentrations on polymer formation. We performed in vitro polymerization by varying the amount of $\mathrm{H}_{2} \mathrm{O}_{2}$ with respect to ETE-S while keeping constant the HRP concentration to $5 \mathrm{U} \mathrm{ml}^{-1}$ (Fig. 3a). The solution was allowed to react for 24 hours before acquiring the UV-Vis spectra. We observe that for $\mathrm{H}_{2} \mathrm{O}_{2} /$ ETE-S $<\sim 0.5, \mathrm{H}_{2} \mathrm{O}_{2}$ is a limiting factor for the reaction as not all ETE-S is polymerized, while for $\mathrm{H}_{2} \mathrm{O}_{2}$ /ETE-S $>0.5$, all ETE-S polymerizes (Fig. 3b). Therefore, at lower $\mathrm{H}_{2} \mathrm{O}_{2}$ concentration, insufficient radicals are formed, thus limiting the polymerization. By increasing the concentration of $\mathrm{H}_{2} \mathrm{O}_{2}$ we reach an optimal point where all ETE-S is polymerized. This indicates that the $\mathrm{H}_{2} \mathrm{O}_{2}$ concentration is the limiting factor for the polymerization reaction suggesting that this could be the origin of the incomplete polymerization observed in the plant extract (Fig. 1b), although other factors cannot be excluded.

Next, we examined how the enzyme concentration is influencing the polymerization reaction. By keeping the $\mathrm{H}_{2} \mathrm{O}_{2}$ /ETE-S molar ratio equal to the optimal value, being 0.5 , we varied the enzyme concentration. The UV-Vis spectra were taken after 1 hour of reaction (Fig. 3c). For the HRP activity above $1 \mathrm{U} \mathrm{ml}^{-1}$, the reaction is completed within $5 \mathrm{~min}$, while for $\mathrm{HRP}=0.1 \mathrm{U} \mathrm{ml}^{-1}$ the reaction is completed after $30 \mathrm{~min}$ (Fig. S3, ESI $\dagger$ ). Apart from influencing the reaction kinetics, HRP also affects the polymerization product. For HRP $\geq 25 \mathrm{U} \mathrm{ml}^{-1}$ we observe a blue shift in the absorption peak of the polymer (Fig. 3d). This can be explained by the formation of shorter oligomeric chains in high enzyme concentrations. As we described, the polymerization occurs by combining ETE-S radicals or by radical transfer to oligomeric units. At high HRP concentrations, more ETE-S radicals will be formed resulting in a higher probability of radical coupling and the formation of oligomers. While at lower HRP concentrations less ETE-S radicals will be formed, therefore the polymerization is likely to happen with radical transfer to polymeric chains rather than radical monomer coupling. ${ }^{28}$ The shift in the absorption spectra could also be a result of polymer aggregation.

To further confirm our findings of the involvement of HRP and $\mathrm{H}_{2} \mathrm{O}_{2}$ in the in vivo polymerization, we functionalized plant roots with ETE-S by modifying the protocol that was developed for the functionalization of the vascular system in roses. ${ }^{6}$ Plant roots do not have a cuticle and the epidermal cells are therefore exposed, allowing the polymerization to be present directly on their surface. We choose bean plants as our model system. A root of an intact bean plant was immersed into a $1 \mathrm{mg} \mathrm{ml} \mathrm{ml}^{-1}$ aqueous solution of ETE-S (1.8 mM) of pH 5-6. After 24 hours of incubation, the root was completely covered with the polymer, Fig. $4 \mathrm{a}$.

At the same time, we stained roots with Amplex Red, a dye that under a peroxidase/peroxide reaction converts into resorufin, 
a.

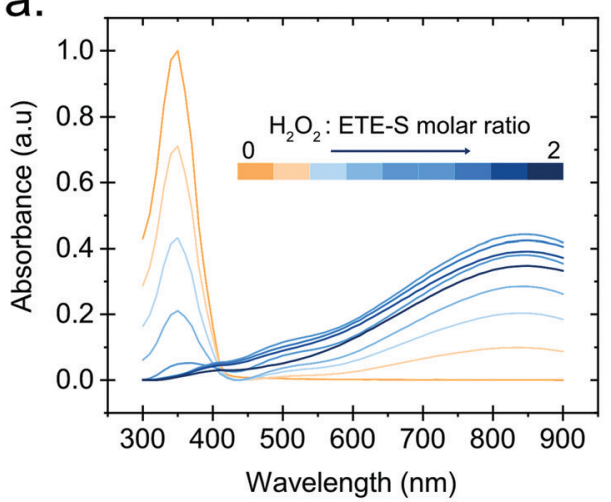

C.

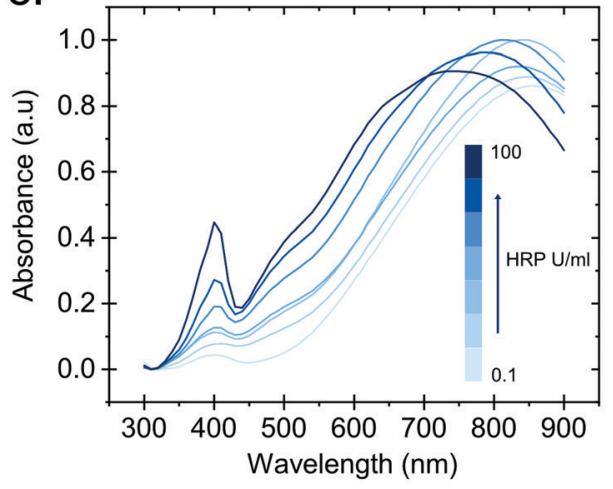

b.

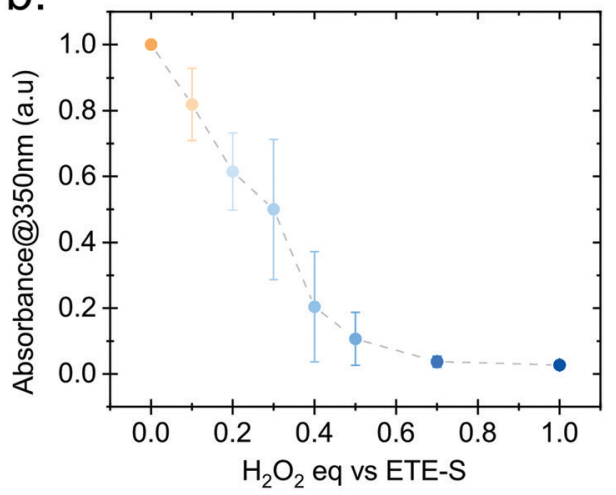

d.

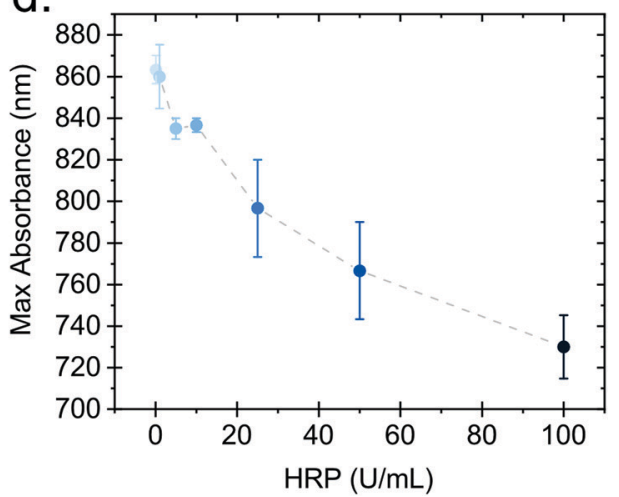

Fig. 3 Effect of $\mathrm{H}_{2} \mathrm{O}_{2}$ and $\mathrm{HRP}$ concentration on the ETE-S in vitro polymerization. (a) Absorption spectra of $\mathrm{p}(\mathrm{ETE}-\mathrm{S})$ formed in vitro with $5 \mathrm{U} \mathrm{ml}^{-1} \mathrm{HRP}$ and various molar equivalents of $\mathrm{H}_{2} \mathrm{O}_{2}$ to ETE-S. (b) Absorbance at $350 \mathrm{~nm}$ for the spectra shown in (a) as a function of the molar equivalents of $\mathrm{H}_{2} \mathrm{O}_{2}$ to ETE-S. (c) Absorption spectra of $\mathrm{p}(\mathrm{ETE}-\mathrm{S})$ formed in vitro for various HRP concentrations. (d) Wavelength corresponding to the highest absorbance value in (c) as a function of the HRP concentration. For (b) and (d) $n=3$.

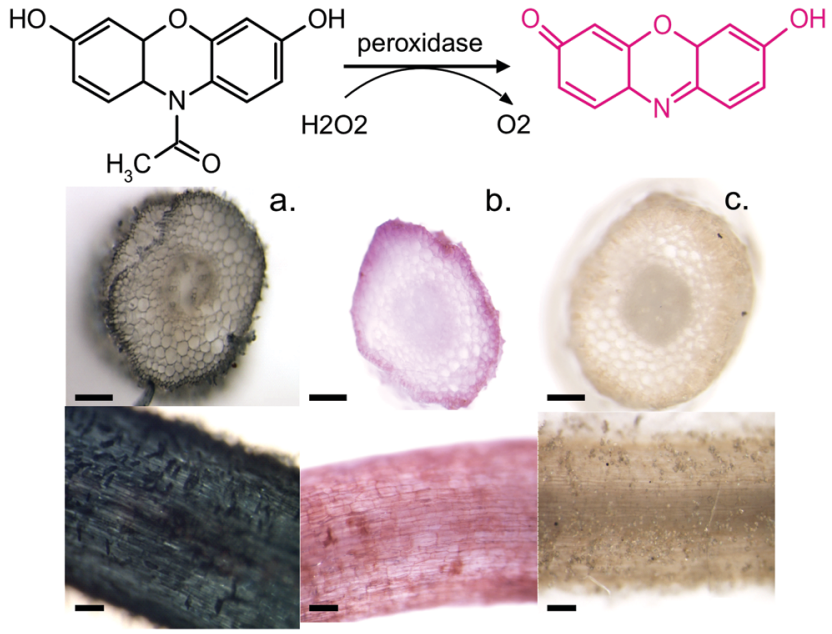

Fig. 4 Amplex Red conversion to resorufin in the presence of HRP and $\mathrm{H}_{2} \mathrm{O}_{2}$. Top view and cross section of (a) bean root after 24 hours in $1 \mathrm{mg} \mathrm{ml}^{-1}$ ETE-S. (b) Bean root after Amplex Red staining. (c) Boiled bean root after Amplex Red staining. Scalebar represents $100 \mu \mathrm{m}$.

a molecule with an intense red color. Fig. $4 \mathrm{~b}$ shows roots after staining with the Amplex Red for 3 hours. It can be seen that the roots acquire a red coloration on the epidermis and around cell walls. The staining covers the whole epidermis while its presence around the cell wall is more intense. To confirm that the coloration occurs only when both $\mathrm{HRP}$ and $\mathrm{H}_{2} \mathrm{O}_{2}$ are present, we boiled a root in water for $30 \mathrm{~min}$ to denature the enzymes. Indeed, Fig. 4c shows that the boiled roots do not acquire any color. Even if we add $\mathrm{H}_{2} \mathrm{O}_{2}$ at the boiled roots, no coloration is observed (Fig. S4, ESI $\dagger$ ) because the Amplex Red requires the presence of both $\mathrm{H}_{2} \mathrm{O}_{2}$ and the active peroxidase. From Fig. 4, we observe that the ETE-S polymer and Amplex Red staining are localized in the same areas of the plant suggesting that the polymerization occurs where both $\mathrm{H}_{2} \mathrm{O}_{2}$ and HRP are present simultaneously.

Furthermore, we performed fluorescence microscopy to observe the polymer on the functionalized cross section of the root (Fig. 5). We used two filter sets, DAPI $(337 \mathrm{~nm}$, $447 \mathrm{em})$ and FTIC $(482 \mathrm{~nm}, 536 \mathrm{~nm})$ that correspond to the spectra of the ETE-S and the neutral p(ETE-S). ${ }^{6}$ Nonfunctionalized cross sections exhibit auto fluorescence while functionalized roots do not exhibit any fluorescence, in either of the two filter sets tested. However, the cross sections appear to be darker in the fluorescence mode at the areas where polymerization occurred. This indicates that the polymer is in its oxidized state and that the ETE-S molecule has polymerized. From both the brightfield images as well as the fluorescent images, it is clear that the polymerization is localized around the cell wall. 


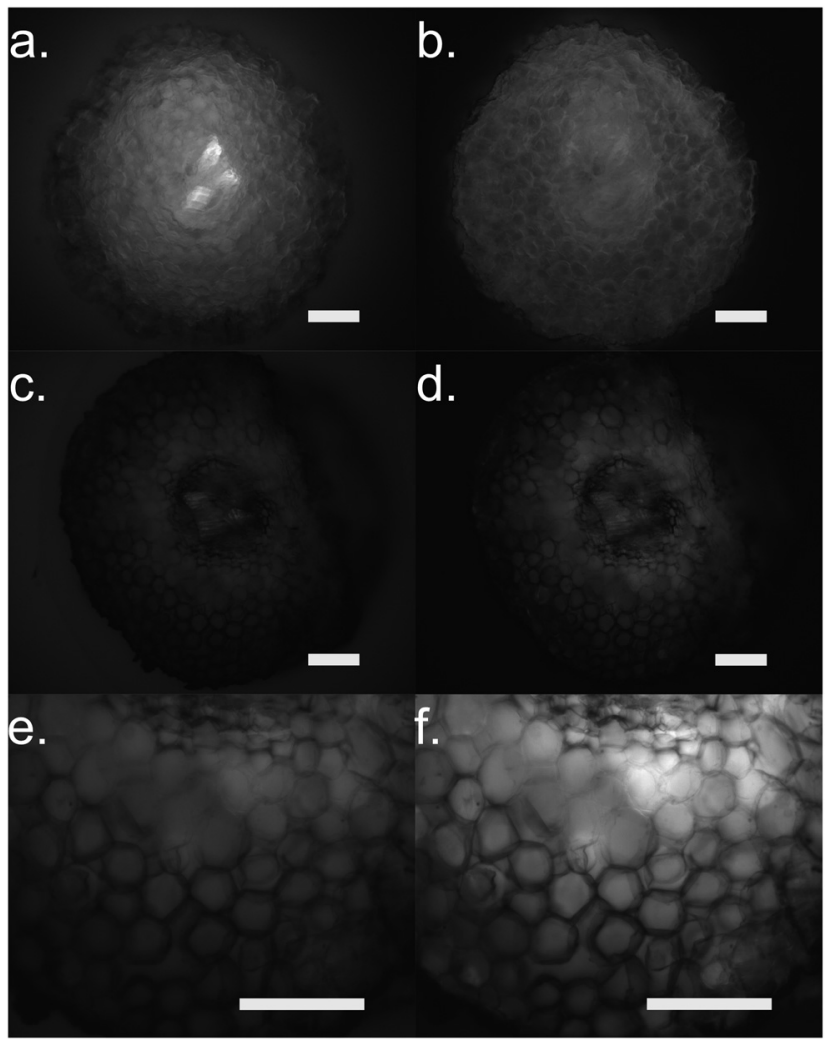

Fig. 5 Fluorescence microscopy of root cross sections after functionalization with ETE-S, $1 \mathrm{mg} \mathrm{ml}^{-1}$ for 2 hours. (a), (c) and (e) DAPI mode for (a) control root and (c and e) functionalized root with (c) same exposure settings as control and (e) longer exposure as control. (b), (d) and (f) FTIC mode for (b) control root and ( $d$ and f) functionalized root with (d) same exposure settings as control (f) and longer exposure as control. Scale bar represents $100 \mu \mathrm{m}$.

\section{Conclusions}

Peroxidases are highly localized in the plant cell wall since they are vital for tuning cell wall density with the help of $\mathrm{H}_{2} \mathrm{O}_{2}$. Two distinct catalytic cycles exist - the hydroxylic and peroxidative cycles - responsible for decreasing and increasing the cell wall density, respectively. The peroxidative cycle crosslinks cell wall compounds including the formation of lignin and suberin within the cell wall, forming a physical barrier that stops cell elongation and protects from external aggressors. Our findings suggest that the ETE-S triggers the peroxidative cycle of the plant, enters into the cycle and polymerizes along the cell wall. Why ETE-S triggers the peroxidative cycle of the plant is not yet understood and in order to pinpoint this at the molecular level further studies are needed.

In this work, we elucidate the mechanism of in vivo polymerization of ETE-S. We demonstrate that ETE-S can be polymerized in vitro at physiological $\mathrm{pH}(5-6)$ through enzymatic polymerization catalysed by HRP in the presence of $\mathrm{H}_{2} \mathrm{O}_{2}$. ETE-S has relatively low oxidation potential (Fig. S5, ESI $\dagger$ ) and this can be a reason for the effective enzymatic polymerization. In vivo this polymerization route occurs naturally in the plant cell wall as part of the plant's defence response and lignification process.
Our findings suggest that indeed the ETE-S is localized in the plant cell wall. This work paves the way for rational design of materials that can be distributed into living systems to augment functionality. In the future, by tuning the molecular structure, backbone and side chains, one can target specific binding but also target the polymerization route in the in vivo environment.

\section{Methods}

\section{UV-Vis absorption spectrometry characterization of the enzymatic polymerisation}

A hydrogen peroxide ( $\geq 30 \%$, Sigma Aldrich) stock solution of $10 \mathrm{mM}$ was prepared freshly before mixing with an ETE-S stock solution of $1.8 \mathrm{mM}$. Horseradish Peroxidase type VI-A (Sigma Aldrich) aliquots of $1 \mathrm{mg} \mathrm{m} l^{-1}\left(\geq 250 \mathrm{U} \mathrm{ml}^{-1}\right)$ were freshly prepared before use and added to the mixture of $\mathrm{H}_{2} \mathrm{O}_{2} /$ ETE-S. The final concentrations of the reagents were: ETE-S $360 \mu \mathrm{M}$, $\mathrm{H}_{2} \mathrm{O}_{2} 180 \mu \mathrm{M}$ and HRP $5 \mathrm{U} \mathrm{ml}^{-1}$. The $\mathrm{pH}$ of the solutions prior and after the reaction were between $\mathrm{pH} 5-6$. The absorption spectra were recorded from 300 to $900 \mathrm{~nm}$ (step $10 \mathrm{~nm}$ ) using a microplate reader (BioTek, Synergy $\mathrm{H} 1$ ). For the $\mathrm{H}_{2} \mathrm{O}_{2}$ dependence investigation, different amounts of $\mathrm{H}_{2} \mathrm{O}_{2}$ from 0.1 to 2 equivalent $v$ s. ETE-S concentration of $360 \mu \mathrm{M}$ were added. The plate was mixed for $30 \mathrm{~s}$ and spectra were acquired 24 hours after the preparation of the samples. HRP activity was kept constant at $5 \mathrm{U} \mathrm{ml}^{-1}$. For the HRP dependence investigation, the molar ratio ETE-S/ $\mathrm{H}_{2} \mathrm{O}_{2}$ was kept constant at 0.5 with a concentration of ETE-S at $360 \mu \mathrm{M}$. The HRP activity was varied from $0.1 \mathrm{U} \mathrm{ml}^{-1}$ to $100 \mathrm{U} \mathrm{ml}^{-1}$. The plate was mixed for $30 \mathrm{~s}$ and spectra were acquired 1 hour after the preparation of the samples.

\section{In vivo and in vitro formed $\mathrm{p}$ (ETE-S) extraction and characterization}

The protocol for extraction of p(ETE-S) from the plant xylem tissue is the same as the one described previously. ${ }^{6}$ Briefly Rose cuttings were immersed in $1 \mathrm{mg} \mathrm{ml}^{-1}$ ETE-S aqueous solution for $24 \mathrm{~h}$. The stem was collected and the functionalized xylem was dissected in small pieces. Using a mortar and pestle the functionalized xylem was mechanically crushed in DMSO in order to dissolve the polymer and the resulting solution was collected and analyzed. As a control, the same procedure was done on xylem tissue from stems that did not absorbed ETE-S. The UV-Vis spectra (300-1300 nm, step $1 \mathrm{~nm}$ ) of sample and control were taken using a PerkinElmer Lambda 900 spectrometer. To acquire the optical signature of the polymer formed in vivo the contribution of the control (plant tissue) was subtracted from the sample. In order to compare the spectra with the in vitro synthesized P(ETE-S), the polymer was first let to dry and then dissolved in DMSO after mechanical crashing using a mortar and a pestle. The UV-Vis absorption spectra were acquired using the microplate reader (BioTek, Synergy H1), 300 to $900 \mathrm{~nm}$ (step $10 \mathrm{~nm}$ ).

\section{Growth of bean plants}

Bean (Phaseolus vulgaris) seeds were purchased from Impecta, Sweden. The seeds were inoculated in a $1 / 3 \mathrm{mix}$ commercial 
bleach solution/DI water with constant stirring for $5 \mathrm{~min}$, followed by $3 \times 5 \mathrm{~min}$ of rising in DI water. Seeds were germinated in a MS/MES (Duchefa Biochemie) (0.215 g/0.05 g in $100 \mathrm{ml}$ DI water) medium agar gel $\mathrm{pH}=5.7$ in the dark with a $\mathrm{RH} \sim 60 \%$ and at $\sim 25{ }^{\circ} \mathrm{C}$. After one week of germination, the plants were transferred to a liquid plant growth medium from Plantagen (Flytende Plantenaering), Sweden with the same growth conditions and a 12 hours day/12 hours night cycle. Beans were used after one week of growth.

\section{ETE-S functionalisation of bean roots}

A solution of $1 \mathrm{mg} \mathrm{ml}^{-1}$ ETE-S was prepared in DI water. A root from the bean was isolated and immersed inside an Eppendorf tube for 24 hours while the plant was suspended in a commercial agar medium (SeedThru gel). After 24 hours the root was cut from the bean for further analysis. The $\mathrm{pH}$ of the ETE-S solution prior and after functionalization of the roots remained the same and between 5-6.

\section{Amplex red assay}

Fresh $10 \mathrm{mM}$ aliquots of Amplex Red (Thermofisher) were prepared in DMSO and added to $20 \mathrm{ml}$ DI water to obtain a final concentration of $50 \mu \mathrm{M}$. Fresh roots were cut from a bean plant and incubated in Amplex Red and ETE-S. In order to denaturate the enzymes roots were boiled for $30 \mathrm{~min}$ in water and then were stained with Amplex Red with and without $100 \mu \mathrm{M} \mathrm{H}_{2} \mathrm{O}_{2}$. The samples were then incubated for 3 hours in an opaque Petri dish and then imaged via a Stereomicroscope (Nikon SM Z1500).

\section{Fluorescence microscopy}

Functionalized and non-functionalized roots were cut using a sterile scalpel and deposited on a glass slide. The samples were immobilized under a coverslip using DAKO mounting medium (Agilent) to keep the fluorescence intact and avoid drying. Images were acquired using a fluorescence microscope (fluorescence/water dipping Microscope Nikon Ni-E). DAPI filter (ex: $377 \mathrm{~nm}$ em: $447 \mathrm{~nm}$ ) and FITC filter (ex: $482 \mathrm{~nm}$ em: $536 \mathrm{~nm}$ ) from Semrock. Images in Fig. 5a-d were obtained using an S Plan Fluor ELWD x20(NA:0.45, WD8.2; Nikon) and images in Fig. 5e and $f$ were obtained using a TU Plan ELWD $\times 50$ (NA:0.6, WD11; Nikon) lens.

\section{Conflicts of interest}

There are no conflicts of interest to declare.

\section{Acknowledgements}

The authors wish to thank Roger Gabrielsson (Linköping University) for the synthesis of ETE-S. This work was supported by the European Union's Horizon 2020 research and innovation programme under grant agreement no. 800926 (FET-OPENHyPhOE), by the Swedish Research Council (VR) and the Wallenberg Wood Science Center (KAW 2018.0452) Additional funding was provided by the Knut and Alice Wallenberg Foundation, the Swedish Foundation for Strategic Research (SSF), The European Research Council (ERC) project e-NeuroPharma 834677 and the Swedish Government Strategic Research Area in Materials Science on Advanced Functional Materials at Linköping University (Faculty Grant SFO-Mat-LiU No. 2009-00971). ES thanks L'Oreal-Unesco For Women in Science (FWIS) and TQN thanks the Department of Energy (DE-SC0017659) for the support.

\section{References}

1 D. T. Simon, E. O. Gabrielsson, K. Tybrandt and M. Berggren, Chem. Rev., 2016, 116, 13009.

2 E. Zeglio, A. L. Rutz, T. E. Winkler, G. G. Malliaras and A. Herland, Adv. Mater., 2019, 31, 1806712.

3 B. D. Paulsen, K. Tybrandt, E. Stavrinidou and J. Rivnay, Nat. Mater., 2019, 1.

4 S. M. Richardson-Burns, J. L. Hendricks and D. C. Martin, J. Neural Eng., 2007, 4, DOI: 10.1088/1741-2560/4/2/L02.

5 E. Stavrinidou, R. Gabrielsson, E. Gomez, X. Crispin, O. Nilsson, D. T. Simon and M. Berggren, Sci. Adv., 2015, 1, DOI: $10.1126 /$ sciadv.1501136.

6 E. Stavrinidou, R. Gabrielsson, K. P. R. Nilsson, S. K. Singh, J. F. Franco-Gonzalez, A. V. Volkov, M. P. Jonsson, A. Grimoldi, M. Elgland, I. V. Zozoulenko, D. T. Simon and M. Berggren, Proc. Natl. Acad. Sci. U. S. A., 2017, 114, 2807.

7 S. I. Shoda, H. Uyama, J. I. Kadokawa, S. Kimura and S. Kobayashi, Chem. Rev., 2016, 116, 2307.

8 Z. G. Peter, Soft Matter, 2011, 7, 316.

9 A. V. Karamyshev, S. V. Shleev, O. V. Koroleva, A. I. Yaropolov and I. Y. Sakharov, Enzyme Microb. Technol., 2003, 33, 556.

10 G. V. Otrokhov, O. V. Morozova, I. S. Vasil'eva, G. P. Shumakovich, E. A. Zaitseva, M. E. Khlupova and A. I. Yaropolov, Biochemistry, 2013, 78, 1539.

11 W. Liu, J. Kumar, S. Tripathy, K. J. Senecal and L. Samuelson, J. Am. Chem. Soc., 1999, 121, 71.

12 M. R. Nabid and A. A. Entezami, J. Appl. Polym. Sci., 2004, 94, 254 .

13 V. Rumbau, J. A. Pomposo, A. Eleta, J. Rodriguez, H. Grande, D. Mecerreyes and E. Ochoteco, Biomacromolecules, 2007, 8, 315 .

14 T. Sikora, R. Marcilla, D. Mecerreyes, J. Rodriguez, J. A. Pomposo and E. Ochoteco, J. Polym. Sci., Part A: Polym. Chem., 2009, 47, 306.

15 L. Duan, Y. Zhao, F. Guo, W. Liu, C. Hou and Z. Ni, Polym. Adv. Technol., 2014, 25, 896.

16 S. Nagarajan, J. Kumar, F. F. Bruno, L. A. Samuelson and R. Nagarajan, Macromolecules, 2008, 41, 3049.

17 G. Shumakovich, G. Otrokhov, I. Vasil'Eva, D. Pankratov, O. Morozova and A. Yaropolov, J. Mol. Catal. B: Enzym., 2012, 81, 66.

18 K. Junker, G. Zandomeneghi, Z. Guo, R. Kissner, T. Ishikawa, J. Kohlbrecher and P. Walde, RSC Adv., 2012, 2, 6478.

19 N. C. Veitch, Phytochemistry, 2004, 65, 249. 
20 F. Passardi, C. Cosio, C. Penel and C. Dunand, Plant Cell Rep., 2005, 24, 255.

21 T. Kawano, Plant Cell Rep., 2003, 21, 829.

22 A. V. Volkov, S. K. Singh, E. Stavrinidou, R. Gabrielsson, J. F. Franco-Gonzalez, A. Cruce, W. M. Chen, D. T. Simon, M. Berggren and I. V. Zozoulenko, Adv. Electron. Mater., 2017, 3, DOI: 10.1002/aelm.201700096.

23 A. S. Rose, A. R. Bradley, Y. Valasatava, J. M. Duarte, A. Prlic and P. W. Rose, Bioinformatics, 2018, 34, 3755, PBD ID: 1GZA.

24 K. Fukuyama, K. Sato, H. Itakura, S. Takahashi and T. Hosoya, J. Biol. Chem., 1997, 272, 5752.
25 B. Yurash, D. X. Cao, V. V. Brus, D. Leifert, M. Wang, A. Dixon, M. Seifrid, A. E. Mansour, D. Lungwitz, T. Liu, P. J. Santiago, K. R. Graham, N. Koch, G. C. Bazan and T.-Q. Nguyen, Nat. Mater., 2019, 1.

26 C.-K. Mai, H. Zhou, Y. Zhang, Z. B. Henson, T.-Q. Nguyen, A. J. Heeger and G. C. Bazan, Angew. Chem., Int. Ed., 2013, 52, 12874.

27 F. Passardi, C. Penel and C. Dunand, Trends Plant Sci., 2004, 9, 534.

28 K. Ryu, J. P. McEldoon, A. R. Pokora, W. Cyrus and J. S. Dordick, Biotechnol. Bioeng., 1993, 42, 807. 